\title{
Species, antibiotic susceptibility profiles and van gene frequencies among enterococci isolated from patients at Mulago National Referral Hospital in Kampala, Uganda
}

David P. Kateete ${ }^{1 *+}$ D, Moses Edolu ${ }^{1,2,3}$, Edgar Kigozi $^{1,3}$, Jeffrey Kisukye ${ }^{3}$, Hannington Baluku ${ }^{3}$, Frank Nobert Mwiine ${ }^{2}$ and Christine F. Najjuka ${ }^{3^{*}+}$

\begin{abstract}
Background: The increase in drug resistance to affordable antibiotics used to treat Gram positive bacterial infections has complicated the management of enterococcal infections. Resistance to vancomycin, one of the most powerful antibiotics, is of utmost concern as both intrinsic and acquired forms of resistance do occur in enterococci. This crosssectional study aimed to determine the species, antibiotic susceptibility profiles and vanA/vanB gene frequencies among enterococci isolated from patients at Mulago Hospital in Kampala, Uganda.

Methods: Between November 2011 and October 2012, stool, urine, sputum and blood samples, as well as vaginal, endocervical, pus, ear and urethra swabs from 3229 patients were processed for isolation of bacteria, yielding 162 enterococci of which 115 were available for analysis (one isolate per specimen/patient). Species-level confirmation and susceptibility testing were determined with the Phoenix ${ }^{\mathrm{TM}}$ AST/ID Automated System, while vanA/ vanB gene carriage was determined by PCR.

Results: Species-level identification revealed 72 isolates of E. faecalis, 20 E. gallinarum/casseliflavus, 5 E. faecium, 4 E. raffinosus and 2 isolates each for $E$. hirae and $E$. durans. Ten isolates could not be identified to species level. Antibiotic resistance was generally low especially to ampicillin, quinolones, nitrofurantoin, glycopeptides and linezolid, but high for erythromycin and tetracycline. Equally, vanA and vanB gene frequencies were low (i.e. 15.8 and 7.9\%, respectively) and detected only in E. casseliflavus/gallinarum species that are intrinsically resistant to vancomycin. Vancomycin resistant isolates of $E$. faecalis and $E$. faecium were not detected.
\end{abstract}

(Continued on next page)

\footnotetext{
* Correspondence: dkateete@chs.mak.ac.ug; cnajjuka@chs.mak.ac.ug

${ }^{\dagger}$ David P. Kateete and Christine F. Najjuka contributed equally to this work.

${ }^{1}$ Department of Immunology and Molecular Biology, Makerere University

College of Health Sciences, Kampala, Uganda

${ }^{3}$ Department of Medical Microbiology, Makerere University College of Health

Sciences, Kampala, Uganda

Full list of author information is available at the end of the article
}

(c) The Author(s). 2019 Open Access This article is distributed under the terms of the Creative Commons Attribution 4.0 International License (http://creativecommons.org/licenses/by/4.0/), which permits unrestricted use, distribution, and reproduction in any medium, provided you give appropriate credit to the original author(s) and the source, provide a link to the Creative Commons license, and indicate if changes were made. The Creative Commons Public Domain Dedication waiver (http://creativecommons.org/publicdomain/zero/1.0/) applies to the data made available in this article, unless otherwise stated. 
(Continued from previous page)

Conclusions: Enterococcus species are frequent in clinical specimens at Mulago Hospital but they are highly susceptible to common antibiotics especially ampicillin. While vancomycin resistant enterococcal (VRE) isolates of $E$. faecium and $E$. faecalis are rare in the hospital and frequency of multidrug resistance is low, non-faecium and non-faecalis VRE isolates (i.e. E. gallinarum/casseliflavus) are frequent, some with VanANanB (high-level) vancomycin resistance. Therefore, species-level identification of enterococci is necessary in resource limited settings to guide infection control and treatment of enterococcal infections.

Keywords: Enterococcus, Enterococcus faecium, Enterococcus faecalis, Vancomyicin resistant enterococci, VRE, vanA, vanB, Mulago hospital, Kampala-Uganda

\section{Background}

The enterococci are a diverse and versatile group of Gram positive, lactic acid producing bacteria, of which Enterococcus faecalis and E. faecium are the most predominant species. While they primarily inhabit the gastrointestinal tract of humans and other mammals, enterococci are also widely distributed in nature: they can be found in soil, water, dairy products and other foodstuffs, and on plants $[1,2]$. They are able to thrive under a variety of conditions and this creates a constant source of infection. Because of their inherent and acquired antibiotic resistance mechanisms, and ability to survive in harsh environments in community and hospital settings [3], enterococci have emerged as major causes of difficult-to-treat nosocomial and community acquired infections particularly biliary and urinary tract infections, bacteremia, endocarditis, intra-abdominal infections, pelvic infections, etc.

As mentioned, enterococci are intrinsically resistant to a variety of antibiotics especially cephalosporins, sulfonamides, oxacillin, ertapenem and perfloxacin. Specifically, E. faecalis and E. faecium are intrinsically resistant to cephalosporins, aminoglycosides, clindamycin, trimethoprim-sulfamethoxazole and fusidic acid; yet again $E$. faecalis is intrinsically resistant to quinupritin. Moreover, through mutation and horizontal gene transfer (HGT) processes, enterococci are able to acquire additional resistance mechanisms to key antibiotics notably tetracycline, erythromycin, fluoroquinolones, rifampicin, chloramphenicol, nitrofurantoin, fusidic acid, glycopeptides (vancomycin \& teicoplanin) and to high concentrations of aminoglycosides and $\beta$-lactams [4]. While $E$. gallinarum and E. casseliflavus are less pathogenic than $E$. faecalis and E. faecium and they are highly susceptible to ampicillin, they are intrinsically resistant to one of the most potent antibiotics, vancomycin [4-6]. Furthermore, the hospitalization of patients and progress in medical technology and treatment, as well as increase in antibiotic usage, have contributed to a surge in infections due to multidrug resistant (MDR) enterococci and vancomycin resistant enterococci (VRE). VRE are of utmost concern in that vancomycin is a powerful antibiotic used to treat
Gram positive bacterial infections yet, both intrinsic and acquired forms of resistance do occur in enterococci. VRE have become an important cause of serious invasive infections globally to such an extent that clinical microbiology laboratories are encouraged to speciate enterococcal isolates from hospitals and screen them for vancomycin resistance $[4,6]$.

Glycopeptide resistance in enterococci is of two main phenotypes $[4,6]$. The first and most common vancomycin resistance phenotype among VRE is high-level resistance that occurs through acquisition of $v a n A$ \& vanB genes, usually in E. faecium and E. faecalis, the species comprising majority of enterococcal infections especially bloodstream/invasive VRE infections. Intrinsic resistance to vancomycin also occurs in enterococci, characteristically in E. gallinarum and E. casseliflavus. These species possess the VanC enzymes, which synthesize pentapeptide peptidoglycan precursors ending in D-alanyl-Dserine that have reduced affinity for vancomycin hence, they are the source of intrinsic vancomycin resistance in E. gallinarum/casseliflavus $[4,6]$. Note, high-level vancomycin resistance of the VanA/VanB phenotype is transferrable and it occurs in E. gallinarum/casseliflavus [4-6]. Overall, VRE other than E. faecium and E. faecalis are infrequently associated with infections but their frequencies are likely to be underreported in the developing countries.

Henceforth, it has become necessary to screen enterococci isolated from hospitalized patients for vancomycin resistance, especially isolates from patients with endocarditis and meningitis, and isolates from sterile body parts [4]. VRE speciation is particularly important as it allows differentiation of $v a n C$ carrying organisms and avoid misidentifying them as VanA/VanB VRE. Furthermore, as colonization of the gut and other body parts by the enterococci is a key predisposing factor for infection and it is the main source of endogenous infection by enterococci [2], characterizing enterococci from clinical specimens is proxy for understanding enterococcal colonization, and it generates insight into their infection dynamics in specific settings.

Although enterococci are amongst the leading causes of urinary tract infections (UTIs) and bacteremia worldwide 
[7], their significance in Uganda is not clear as there are no clearly defined rates for enterococcal infections in this country. All the same, preliminary estimates for enterococcal infections reveal high rates for UTIs and surgical site infections (SSIs) in Uganda [8, 9]. The aim of this study was to define the species and antibiotic susceptibility profiles of enterococci isolated from patients at Mulago National Referral Hospital in Kampala, Uganda. We also estimated the $v a n A$ and $v a n B$ gene frequencies among the isolates.

\section{Methods}

\section{Study setting, specimens and isolates}

This was a cross-sectional study conducted at the Clinical Microbiology Laboratory of the Department of Medical Microbiology, Makerere University College of Health Sciences. The molecular assays were performed at the Molecular Diagnostics Laboratory of the Department of Immunology \& Molecular Biology, Makerere University College of Health Sciences. Between December 2011 and November 2012, the Clinical Microbiology Laboratory processed 3229 clinical specimens from approx. The same number of patients (i.e. one specimen per patient) at Mulago National Referral Hospital, for isolation of bacteria. The specimens processed included blood 34\% (1094/3229), sputum 24\% (777/3229), high vaginal swabs 9\% (287/3229), urine 7.8\% (252/3229), endocervical swabs $7.8 \%$ (252/3229), stool 7.4\% (238/3229), pus swabs $4.1 \%$ (133/3229), ear swabs 1.3\% (42/3229), urethra swabs $1.3 \%$ (42/3229), cerebrospinal fluid 1.3\% (42/3229), lymph node aspirates $0.65 \%$ (21/3229), oral swabs $0.43 \%$ (14/3229), rectal swabs $0.22 \%$ (7/3229), tracheal aspirates $0.22 \%$ (7/3229), broncho-alveolar lavage $0.22 \%$ (7/3229), pharyngeal swabs $0.22 \%$ (7/3229) and pleural fluids $0.22 \%$ (7/3229), Additional file 1: Table S1. These specimens were processed on request by clinicians during routine clinical investigations.

Of the 3229 specimens, Gram positive and catalase negative isolates grew from a total of 196 specimens (one isolate per specimen). All the 196 Gram positive and catalase negative bacterial isolates had a diplococcal chain appearance under microscopy, and they were subjected to growth on bile esculin agar (BEA), a selective differential medium used to identify members of the genus Enterococcus. Additionally, a 6.5\% (w/v) sodium chloride tolerance test was carried out in order to differentiate enterococci from group D streptococci. Following these two procedures, a total of 162 pure isolates (one per specimen) were presumptively confirmed to be enterococci and stored at $-80^{\circ} \mathrm{C}$.

Presumptive species-level identification was based on motility and pigmentation, and formation of acid in mannitol, sorbitol, sucrose, arabinose and pyruvate broths [6]. Briefly for the pyruvic acid test, pyruvate broth was inoculated with a single colony from a $24 \mathrm{~h}$ pure culture, incubated aerobically at $35^{\circ} \mathrm{C}$ with a loose cap and examined daily for 3-5 days. Acid production was confirmed by color change in the bromothymol indicator from blue-green to yellow, which was interpreted as positive for E. faecalis. E. faecalis ATCC51299 was used as the positive control for the pyruvate test. For the arabinose test, Brain Heart Infusion (BHI) broth containing arabinose and bromocresol purple indicator was inoculated with a single colony from a $24 \mathrm{~h}$ pure culture, and incubated overnight at $35^{\circ} \mathrm{C}$. Color change in the media to yellow was interpreted as positive for $E$. faecium [10]. For motility test, microscopy was done to presumptively differentiate $E$. gallinarum from other enterococci.

Species confirmation and antimicrobial susceptibility testing Species-level confirmation and antimicrobial susceptibility testing (AST) by minimum inhibitory concentration (MIC) were achieved by the BD Phoenix ${ }^{\mathrm{TM}}$ Automated Identification \& Susceptibility Testing system according to the manufacturer's recommendations. The BD Phoenix $^{\mathrm{TM}}$ default MIC breakpoints to a panel of 11 antibiotics to which enterococci are susceptible were used: ampicillin $\leq 2 \mu \mathrm{g} / \mathrm{ml}$, daptomycin $4 \mu \mathrm{g} / \mathrm{ml}$, teicoplanin $\leq 1 \mu \mathrm{g} / \mathrm{ml}$, vancomycin $\leq 0.5 \mu \mathrm{g} / \mathrm{ml}$, erythromycin $\leq 0.25 \mu \mathrm{g} / \mathrm{ml}$, linezolid $\leq 1 \mu \mathrm{g} / \mathrm{ml}$, nitrofurantoin $64 \mu \mathrm{g} / \mathrm{ml}$, ciprofloxacin $\leq 0.5 \mu \mathrm{g} / \mathrm{ml}$, moxifloxacin $\leq 0.5 \mu \mathrm{g} / \mathrm{ml}$, tetracycline $\leq 0.5 \mu \mathrm{g} /$ $\mathrm{ml}$, and gentamicin-syn $\leq 500 \mu \mathrm{g} / \mathrm{ml}$. For quality control, $E$. faecalis ATCC29212 and E. faecium HA56038 (ATCC) were included in the Phoenix AST \& ID panels. Figure 1 depicts the diagnostic strategy we used to identify enterococci to genus and species levels.

\section{Genotyping}

To detect the $v a n A$ and $v a n B$ genes in enterococci, PCR was performed on a Techne TC-412 thermocyler in reaction volumes of $12.5 \mu \mathrm{l}$ using previously published primers [11] 5' -AATGTGCGAAAAACCTTGCG-3' \& 5' -CCGTTTCCTGTATCCGTCC-3' (for vanA); 5' -CA AATCACTGGCCTACATTC-3' \& 5' -TCTGCATCCAA GCACCCG-3' (for $v a n B$ ). Each reaction contained $5 \mu \mathrm{l}$ of $2 x$ Taq master mix (Sigma Co.), $1 \mu \mathrm{l}$ of forward and reverse primers, $3 \mu \mathrm{l}$ nuclease free water and $2.5 \mu \mathrm{l}$ of crude enterococcal DNA extract (template). The cycling conditions were as follows: Initial denaturation at $94^{\circ} \mathrm{C}$, 4 min followed by 31 cycles of $94^{\circ} \mathrm{C} 1 \mathrm{~min}, 50{ }^{\circ} \mathrm{C} 1 \mathrm{~min}$, and $68^{\circ} \mathrm{C} 1 \mathrm{~min}$, and a final extension step at $68^{\circ} \mathrm{C}, 10$ min. The PCR products were analyzed by agarose gel electrophoresis with ethidium bromide staining $(0.5 \mu \mathrm{g} /$ $\mathrm{ml}$ ). Repetitive elements-based PCR (Rep-PCR) genotyping for insight into genetic relatedness of the isolates was performed as previously described [12]. 


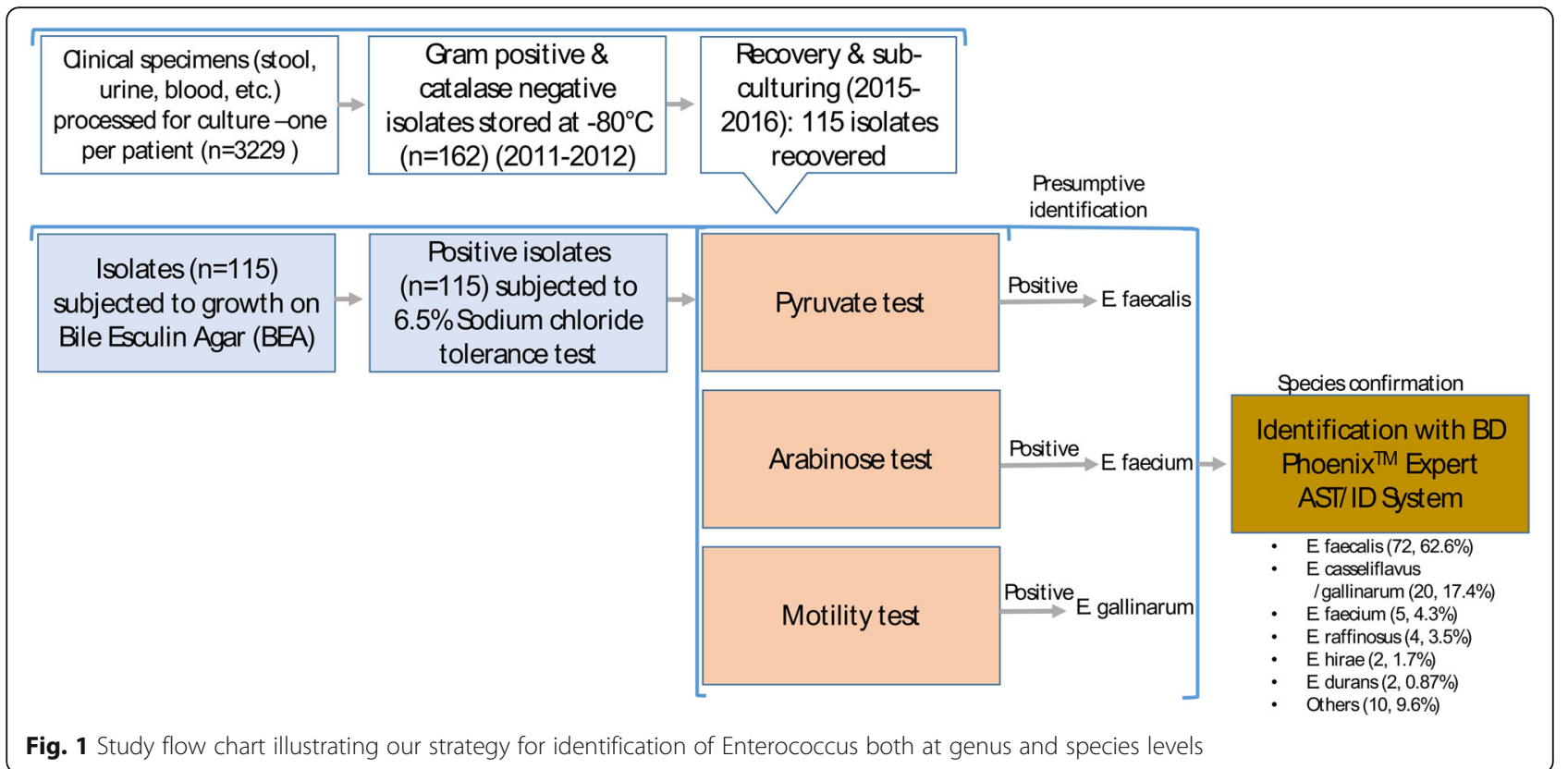

\section{Results}

Frequency of Enterococcus species

We processed a total of 3229 specimens from 3229 patients, yielding 162 Gram positive and catalase negative isolates (one isolate per patient/specimen), of which 115 were available for analysis. Females were 2066 (64\%) and the median age of the patients with samples yielding enterococci was 28 years (Interquartile Range (IQR) of 19 to 44). All the 115 isolates grew on BEA and survived in BHI broth supplemented with $6.5 \%$ sodium chloride hence, they were presumptively identified as enterococci, Fig. 1. Therefore, the overall frequency of enterococci in the processed clinical samples was $17.6 \%(42 / 238)$ stool, $10 \%(25 / 252)$ urine, $1.9 \%(21 / 1094)$ blood, $1.93 \%(15 /$ $777)$ sputum, $7.5 \%(10 / 133)$ pus swabs and $4.8 \%(02 / 42)$ ear swabs. The $47(29 \%, 47 / 162)$ stored isolates that were not recovered on sub-culturing were mainly from vaginal swabs (27), urine (7), stool (3), endo-cervical swabs (2), urethra swabs (7) and rectal swabs (1), Additional file 1: Table S1. Once more, there was no enterococcus growth from cerebrospinal fluids, lymph node aspirates, tracheal aspirates, bronchoalveolar lavage, pleural fluids, oral and pharyngeal swabs, Additional file 1: Table S1. Species-level identification of the enterococcus with the BD Phoenix ${ }^{\mathrm{mm}}$ AST/ID system yielded 72 isolates of E. faecalis, 20 E. gallinarum/casseliflavus, 5 E. faecium, 4 E. raffinosus, and 2 isolates each for $E$. hirae and $E$. durans. Ten isolates could not be identified to species level, Table 1.

Antibiotic susceptibility profiles and van gene frequencies Antibiotic resistance was generally low for all the species especially to ampicillin, quinolones (ciprofloxacin \& moxifloxacin), gentamicin-syn, nitrofurantoin, glycopeptides, linezolid and daptomycin but high for erythromycin and tetracycline, Table 2. All E. faecalis isolates were susceptible to nitrofurantoin, vancomycin, teicoplanin and linezolid but resistant to erythromycin and tetracycline, Table 2. Further, all but one isolate of $E$. faecalis that were intermediate or resistant to ciprofloxacin were also resistant to tetracycline, and all except two were resistant to erythromycin. Of the three isolates that were resistant to gentamicin-syn, two were E. faecalis (Table 2) one of which exhibited multiple resistance to several antibiotics (i.e. ampicillin, erythromycin, nitrofurantoin, ciprofloxacin, tetracycline) but susceptible to teicoplanin, vancomycin and daptomycin. For E. faecium, all the five isolates were susceptible to gentamicin-syn, ampicillin, vancomycin, teicoplanin, linezolid and moxifloxacin however, four were non-susceptible to daptomycin. One isolate of E. faecium was resistant to both erythromycin and nitrofurantoin and intermediate to ciprofloxacin.

Table 1 Enterococcus isolates identified to species level

\begin{tabular}{lll}
\hline Species & Number of isolates $^{\text {a }}$ & Percent $^{\text {Enterococcus faecalis }}$ \\
\hline Enterococcus casseliflavus / gallinarum & 20 & 62.6 \\
Enterococcus faecium & 05 & 17.4 \\
Enterococcus raffinosus & 04 & 4.3 \\
Enterococcus hirae & 02 & 3.5 \\
Enterococcus durans & 02 & 1.7 \\
Others & 10 & 0.87 \\
Total & 115 & 9.6 \\
\hline
\end{tabular}

${ }^{\mathrm{a}}$ One isolate per specimen 
Table 2 Antimicrobial susceptibility profiles of enterococcal isolates investigated

\begin{tabular}{|c|c|c|c|c|c|c|c|c|c|}
\hline \multirow[t]{2}{*}{ Drug } & \multicolumn{3}{|c|}{ E. faecalis, $n=72$} & \multicolumn{3}{|c|}{ E. faecium, $n=05$} & \multicolumn{3}{|c|}{ Others, $n=38$} \\
\hline & R (\%) & I (\%) & S (\%) & R (\%) & I (\%) & S (\%) & R (\%) & I (\%) & S (\%) \\
\hline Erythromycin & $54(72)$ & $07(9.7)$ & $11(15.3)$ & $04(80)$ & 0 & $01(20)$ & $07(18.4)$ & $04(10.5)$ & $11(28.9)$ \\
\hline Tetracycline & $50(69.4)$ & 0 & $22(30.6)$ & $03(60)$ & 0 & $02(40)$ & 05 (13.2) & 0 & $33(86.8)$ \\
\hline Ciprofloxacin & $07(9.7)$ & $06(8.3)$ & 59 (81.9) & $03(60)$ & $01(20)$ & $01(20)$ & $x$ & $x$ & $x$ \\
\hline Moxifloxacin & $01(0.14)$ & 0 & 71 (98.6) & 0 & 0 & $05(100)$ & $04(10.5)$ & $04(10.5)$ & $30(78.9)$ \\
\hline Ampicillin & $01(1.4)$ & 0 & 71 (98.6) & 0 & 0 & $05(100)$ & $02(5.3)$ & 0 & $36(94.7)$ \\
\hline Gentamicin-Syn & $02(2.8)$ & 0 & $72(100)$ & 0 & 0 & 05 (100) & $01(2.6)$ & 0 & $01(2.6)$ \\
\hline Nitrofurantoin & 0 & 0 & $72(100)$ & $01(20)$ & $01(20)$ & $03(60)$ & $01(2.6)$ & $03(7.9)$ & $34(89.5)$ \\
\hline Vancomycin & 0 & 0 & $72(100)$ & 0 & 0 & 05 (100) & $20(52.6)$ & 0 & $18(47.4)$ \\
\hline Teicoplanin & 0 & 0 & $72(100)$ & 0 & 0 & $05(100)$ & $03(7.9)$ & 0 & $35(92.1)$ \\
\hline Linezolid & 0 & 0 & $72(100)$ & 0 & 0 & $05(100)$ & $01(2.6)$ & $04(10.5)$ & $34(89.5)$ \\
\hline Daptomycin & 0 & 0 & $64(88.9)$ & 0 & 0 & $01(20)$ & 0 & 0 & 07 (18.4) \\
\hline MDR & & NA & NA & & NA & NA & & NA & NA \\
\hline $\operatorname{van} A$ & - & - & - & - & - & - & $06(15.8)$ & - & - \\
\hline $\operatorname{van} B$ & - & - & - & - & - & - & $03(7.9)$ & - & - \\
\hline
\end{tabular}

$S$ Susceptible, I Intermediate, $R$ Resistant; -,Negative; $\mathrm{x}-\mathrm{MICS}$ for these species (see "others") are not reported by the Phoenix system MDR, multidrug resistant (isolate resistant to three or more antibiotic classes)

"Others" denotes E. casseliflavus/gallinarum, E. raffinosus, E. hirae, E. durans, and the 10 isolates that were not identified to species level

Furthermore, all the other species combined (i.e. $E$. casseliflavus/gallinarum, E. raffinosus, E. hirae, E. durans and the 10 isolates that were not identified to species level) exhibited varied susceptibilities to antimicrobials with a total of 31 isolates being non-susceptible to daptomycin. Overall, vancomycin resistance in this study was detected only in the 20 isolates of E. casseliflavus/ gallinarum (Table 2) that are intrinsically resistant to this drug. Likewise, resistance to teicoplanin was detected in only E. casseliflavus/gallinarum at $15 \%(3 / 20)$; however, the teicoplanin resistant isolates were susceptible to ampicillin, erythromycin, nitrofurantoin, tetracycline and gentamicin-syn. Once more, the only linezolid resistant isolate was E. casseliflavus/gallinarum; it was also resistant to vancomycin, teicoplanin and moxifloxacin but susceptible to ampicillin, tetracycline, nitrofurantoin and gentamicin-syn.

\section{van gene frequencies and multidrug resistance phenotype}

Generally the $v a n A$ and $v a n B$ gene frequencies were low i.e. 15.8 and $7.9 \%$ respectively, and detected in only $E$. casseliflavus/gallinarum species, Table 3 \& Additional file 2: Figure S1. The species and resistance patterns of enterococci resistant to two or more antibiotics are summarized in Table 4. Regarding resistance to antibiotics to which enterococci are commonly susceptible (i.e. erythromycin, tetracycline, ciprofloxacin, moxifloxacin, ampicillin, nitrofurantoin, vancomycin, teicoplanin, linezolid, daptomycin), only 11 isolates $(9.6 \%, 11 / 115)$ were MDR and almost all the MDR patterns involved resistance to tetracycline, Table 4. Majority of MDR isolates belonged to E. faecalis (five isolates) and E. casseliflavus/gallinarum (four isolates) while the remaining two isolates were E. raffinosus and E. durans. Besides MDR, the most common resistance pattern among isolates resistant to two or more antibiotics involved resistance to erythromycin and tetracycline, Table 4. Lastly, cluster analysis of the Rep-PCR fingerprints revealed no evidence of clonal spread/transmission of enterococci in the Mulago Hospital setting (not shown).

\section{Discussion}

In this study, one of the most predominant Enterococcus species, E. faecalis [4, 13, 14], was the most frequent at 62.6\%. However E. faecium, another key enterococcus species worldwide especially in hospital settings, was not frequent. The reported low frequency for E. faecium and several other enterococcus species in this study ( $E$. durans, E. hirae and E. raffinosus) is in line with global reports for these species [4] in that up to $80-90 \%$ of the enterococcal infections in humans are attributed to $E$. faecalis, $10-15 \%$ E. faecium and $<5 \%$ combined other species [6]. Note, the proportion of E. faecium in this study was twofold lower than expected. While this could have resulted from a potential sampling bias, E. faecium being significantly less frequent than $E$. faecalis at Mulago Hospital has previously been reported i.e. prevalence of $8.7 \%(2 / 23)$ and $91.3 \%(21 / 23)$ for E. faecium and E. faecalis, respectively [9].

Interestingly, the second most frequent enterococcus species in this study were the E. gallinarum/casseliflavus 
Table 3 Antibiotic susceptibility profiles of vancomycin resistant E. casseliflavus/gallinarum

\begin{tabular}{|c|c|c|c|c|c|c|c|c|c|c|c|c|}
\hline Isolate \# & AMP & GEN & ERY & TET & MXF & NIT & $\mathrm{DAP}^{\mathrm{a}}$ & LIZ & TEC & VAN & $\operatorname{van} A$ & vanB \\
\hline EM0085 & $S$ & $S$ & $\mathrm{R}$ & $R$ & $S$ & $S$ & N & $S$ & $S$ & $R$ & - & - \\
\hline EM0176 & $S$ & S & $\mathrm{R}$ & S & $S$ & I & N & S & S & $R$ & + & - \\
\hline EM3113 & $S$ & $S$ & $\mathrm{R}$ & R & $S$ & S & N & S & S & R & - & - \\
\hline EM0476 & $S$ & S & S & S & I & S & N & S & $\mathrm{R}$ & R & + & - \\
\hline EM2016 & $S$ & S & $\mathrm{R}$ & $\mathrm{R}$ & $S$ & S & S & S & S & R & - & + \\
\hline EM0040 & $S$ & S & S & S & $\mathrm{R}$ & S & N & S & S & R & - & - \\
\hline EM0049 & $S$ & S & S & S & $\mathrm{R}$ & S & N & S & S & R & + & - \\
\hline EM0149 & $S$ & S & S & $S$ & I & S & S & $S$ & S & R & - & - \\
\hline EM0477 & $S$ & S & S & $S$ & I & $S$ & S & I & $\mathrm{R}$ & $R$ & + & - \\
\hline EM1161 & $\mathrm{S}$ & S & $S$ & S & $S$ & S & S & S & S & R & - & + \\
\hline EM0162 & $S$ & S & I & $S$ & $\mathrm{R}$ & S & N & $\mathrm{R}$ & $\mathrm{R}$ & R & + & - \\
\hline EM0074 & $S$ & S & $S$ & S & I & S & S & S & S & R & - & - \\
\hline EM0014 & $S$ & S & S & S & $S$ & S & S & S & S & $R$ & - & - \\
\hline EM0116 & S & S & S & S & S & S & S & S & S & $R$ & - & + \\
\hline EM0022 & $S$ & $S$ & S & S & $S$ & S & $S$ & S & S & $R$ & - & - \\
\hline EM0056 & $\mathrm{S}$ & S & $S$ & $\mathrm{~S}$ & $\mathrm{~S}$ & S & S & S & S & $R$ & - & - \\
\hline EM0099 & $S$ & S & S & S & $S$ & S & N & S & S & R & - & - \\
\hline EM0010 & S & S & S & S & S & S & S & S & S & R & + & - \\
\hline EM0018 & $S$ & S & S & S & $S$ & S & S & S & S & $R$ & - & - \\
\hline EM1009 & $S$ & S & S & $S$ & $S$ & S & S & $S$ & S & R & - & - \\
\hline Total R/+ (\%) & 0 & 0 & $04(20)$ & $03(15)$ & $03(15)$ & 0 & 0 & $01(5)$ & $03(15)$ & $20(100)$ & $06(30)$ & $03(15)$ \\
\hline
\end{tabular}

E. casseliflavus \& Enterococcus gallinarum are intrinsically resistant to vancomycin

$A M P$ ampicillin, VAN vancomycin; ${ }^{a} G E N$ high level gentamicin, ERY erythromycin, TET tetracyclin, MXF moxifloxacin, NIT nitrofurantoin, DAP daptomycin, TEC teicoplanin, LIZ linezolid, S Susceptible, I Intermediate, $R$ Resistant, $N$ non-susceptible; - , Negative; +, Positive

species at $17.4 \%$, which is higher than expected from global reports for these species (i.e. $\leq 2 \%$ ) [5, 6]. Although E. gallinarum/casseliflavus are infrequently isolated from clinical specimens, especially in the developed countries, several reports have implicated them as causes of serious invasive infections $[5,6]$. These species colonize the gut of hospitalized and non-hospitalized people with overall colonization rates ranging from $5.7-12.1 \%$ [6]. The current study, and recent findings from Ethiopia [15] and Sri Lanka [16], suggest that the colonization rate for these species is high in developing countries. However, as we investigated isolates from symptomatic individuals, our findings should not be used to draw inferences on enterococcus colonization in the wider population. The clinical and epidemiological features of cases of E. casseliflavus/ gallinarum bacteremia in the United States were reported $[5,6]$, with underlying conditions such as immunodeficiency, malignancy, receipt of transplant etc. reported in up to $95 \%$ of the patients $[5,6]$. Of all the risk factors investigated only the immunocompromised status was a significant predictor of mortality in patients with E. gallinarum/casseliflavus bacteremia [5]. Perhaps, the rampant poverty in Uganda and immunodeficiency linked to prevalent HIV-infection and malnutrition are factors that could be responsible for high occurrence of E. gallinarum/casseliflavus in patients at Mulago Hospital, though this requires further investigation. Relatedly, the high frequency of $E$. gallinarum/casseliflavus species in this study was comparable to rates from Ethiopia (26.3\% E. gallinarum/ casseliflavus, $17.5 \%$ E. gallinarum and $8.8 \%$ E. casseliflavus) [15]), a low-income country like Uganda with high HIVinfection rates.

Almost all the enterococci in this study were susceptible to ampicillin (only three isolates were resistant) and this is in agreement with the finding that enterococci are generally susceptible to ampicillin [4]. Note, ampicillin resistance in this study was significantly lower than rates from Ethiopia [15] and India [17], but this could be due to differences in methods for drug susceptibity testing e.g. we used MICs by an automated instrument that are generally more efficient compared to the disk diffusion methods used by investigators in Ethiopia [15]. As ampicillin is the drug of choice in treatment of enterococcal infections, the high susceptibility of isolates implies that this cheap antibiotic is effective in treatment of enterococcal infections in Uganda. Only three isolates exhibited high-level resistance to gentamicin, two of which were $E$. faecalis and these are few compared to rates 
Table 4 Species and resistance patterns of enterococci resistant to two or more antibiotics

\begin{tabular}{|c|c|c|c|}
\hline Isolate ID & Species & Resistance pattern & MDR phenotype \\
\hline EM3132 & E. faecalis & $\begin{array}{l}\text { ERY-GEN-AMP- } \\
\text { CIP-MXF-TET }\end{array}$ & Yes \\
\hline EM2591 & E. faecalis & ERY-GEN-TET & Yes \\
\hline EM0082 & E. faecalis & ERY-CIP-TET & Yes \\
\hline EM0028 & E. faecalis & ERY-CIP-TET & Yes \\
\hline EM1239 & E. faecalis & ERY-CIP-TET & Yes \\
\hline EM0057 & E. faecalis & ERY-TET & No \\
\hline EM0055 & E. faecalis & ERY-TET & No \\
\hline EM0270 & E. faecalis & ERY-TET & No \\
\hline EM0098 & E. faecalis & ERY-TET & No \\
\hline EM0056 & E. faecalis & ERY-TET & No \\
\hline EM0066 & E. faecalis & ERY-TET & No \\
\hline EM0073 & E. faecalis & ERY-TET & No \\
\hline EM1196 & E. faecalis & ERY-TET & No \\
\hline EM2591 & E. faecalis & ERY-TET & No \\
\hline EM0216 & E. faecalis & ERY-TET & No \\
\hline EM0141 & E. faecalis & ERY-TET & No \\
\hline EM0011 & E. faecalis & ERY-TET & No \\
\hline EM2970 & E. faecalis & ERY-TET & No \\
\hline EM1023 & E. faecalis & ERY-TET & No \\
\hline EM0033 & E. faecalis & ERY-TET & No \\
\hline EM0142 & E. faecalis & ERY-TET & No \\
\hline EM0333 & E. faecalis & ERY-TET & No \\
\hline EM1333 & E. faecalis & ERY-TET & No \\
\hline EM0297 & E. faecalis & ERY-TET & No \\
\hline EM0016 & E. faecalis & CIP-TET & No \\
\hline EM0087 & E. faecalis & CIP-TET & No \\
\hline EM0175 & E. faecium & ERY-CIP & No \\
\hline EM0154 & E. raffinosus & GEN-ERY-TET & Yes \\
\hline EM0704 & E. durans & ERY-AMP-NIT-MXF-TET & Yes \\
\hline EM0085 & $\begin{array}{l}\text { E. gallinarum/ } \\
\text { casseliflavus }\end{array}$ & $V A N^{*}-E R Y-T E T$ & Yes \\
\hline EM3113 & $\begin{array}{l}\text { E. gallinarum/ } \\
\text { casseliflavus }\end{array}$ & VAN*-ERY-TET & Yes \\
\hline EM2016 & $\begin{array}{l}\text { E. gallinarum/ } \\
\text { casseliflavus }\end{array}$ & VAN*-ERY-TET & Yes \\
\hline EM0162 & $\begin{array}{l}\text { E. gallinarum/ } \\
\text { casseliflavus }\end{array}$ & VAN*-TEC-LIZ-MXF & Yes \\
\hline EM0476 & $\begin{array}{l}\text { E. gallinarum/ } \\
\text { casseliflavus }\end{array}$ & VAN*-TEC & No \\
\hline EM0477 & $\begin{array}{l}\text { E. gallinarum/ } \\
\text { casseliflavus }\end{array}$ & VAN*-TEC & No \\
\hline EM0176 & $\begin{array}{l}\text { E. gallinarum/ } \\
\text { casseliflavus }\end{array}$ & $V A N^{*}-E R Y$ & No \\
\hline EM0040 & $\begin{array}{l}\text { E. gallinarum/ } \\
\text { casseliflavus }\end{array}$ & VAN*-MXF & No \\
\hline
\end{tabular}

Table 4 Species and resistance patterns of enterococci resistant to two or more antibiotics (Continued)

\begin{tabular}{|c|c|c|c|}
\hline Isolate ID & Species & Resistance pattern & MDR phenotype \\
\hline EM0049 & $\begin{array}{l}\text { E. gallinarum/ } \\
\text { casseliflavus }\end{array}$ & VAN*-MXF & No \\
\hline Total MDR (\%) & & & $11(9.6)$ \\
\hline
\end{tabular}

from Ethiopia [15]. At the moment, high-level gentamicin resistance in enterococci may not be a cause for concern in Uganda however, continuous surveillance in hospital settings is necessary to monitor this trend. Further, several isolates in this study were non-susceptible to daptomycin, and this could be attributed to tolerance ("the ability of the organism to survive levels of drugs well in excess of the MIC" [4]), a common finding among clinical isolates of enterococci [4].

All the E. faecalis and E. faecium isolates in this study were susceptible to vancomycin and the only VRE isolates detected were E. gallinarum/casseliflavus species that are inherently resistant to vancomycin. Elsewhere, investigators in Spain who studied the antibiotic resistance of enterococci from healthy breastfed infants also did not detect VRE isolates of E. faecalis and E. faceicum [18]. As the VRE isolates (i.e. E. gallinarum/casseliflavus) in this study were susceptible to ampicillin, this antibiotic should be the drug of choice for treating enterococcal infections in Uganda as increased use of vancomycin may lead to selection of glycopeptide resistant enterococci in a resource-limited setting. It is important to note that lack of VRE isolates of E. faecalis/ faecium in this study may not imply they are rare in Uganda: a VRE isolate of E. faecalis was recovered from bovine milk samples in Kampala [19] and from a patient with a SSI at Mulago Hospital [9].

The $v a n A$ and $v a n B$ genes were not detected in $E$. faecalis and $E$. faecium, which was expected as these genes encode high-level vancomycin resistance [20] that was not detected in E. faecalis/faecium isolates. Yet, vanA/ vanB occurred in E. gallinarum/casseliflavus; although vancomycin resistance in these species is reported to be of the low-level type [4-6], their detection implies that acquisition of the $v a n A / v a n B$ genes occurred, most likely in food animals as vancomycin resistant $E$. faecalis and E. gallinarum/casseliflavus were recovered from bovine clinical specimens [19]. As high-level vancomycin resistance of the VanA and VanB phenotypes is transferrable, and it has been reported in E. gallinarum and $E$. casseliflavus which are part of the normal flora in mammals [4], individuals in Uganda could be at risk of being colonized by such isolates via transmission from animals $[4,6]$. Overall, our data reveals that speciation and 
accurate identification of VRE isolates in a resourcelimited setting is important [4]. Moreover, because $E$. gallinarum/casseliflavus appear more frequent in human specimens [15, 16] and bovine specimens [19] in lowincome countries compared to the developed countries [4-6], species-level identification of enterococci is key for instituting proper infection control measures and timely antibiotic therapy [6].

While the MDR frequency was low in this study, the isolates exhibited unique resistance patterns, for example ERY-GEN-AMP-CIP-MXF-TET (E. faecalis); ERY-AMPNIT-MXF-TET (E. durans); VAN-TEC-LIZ-MXF (E. gallinarum/casseliflavus); VAN-TEC (E. gallinarum/casseliflavus). Thus, we have reported E. gallinarum/casseliflavus isolates that were resistant to both vancomycin and teicoplanin though they were susceptible to ampicillin. Antibiotic stewardship and strengthening of the infection control practices is necessary to prevent acquisition of ampicillin resistance in such strains. Lastly, cluster analysis of the Rep-PCR fingerprints revealed no evidence of clonal spread/transmission of enterococci in the Mulago Hospital setting though we used an inferior approach to infer genetic relatedness among isolates. However, this finding is in accordance with reports that VRE isolates in certain settings (e.g. the United States) are genetically diverse [4].

There were certain limitations in this study: First, a total of 47 stored enterococcal isolates mainly from vaginal swabs, urine and stool were not recovered on subculturing. However, as we succeeded in sub-culturing isolates from specimens where enterococci grew most (see Additional file 1: Table S1), we believe we avoided a potential selection bias. Second, we could not rule-out contamination: Given the high rate of colonization of health care workers by the enterococci, some of the isolates might have been not clinically relevant. Third, we did not investigate E. gallinarum/casseliflavus for vanC genes as these are chromosomally encoded hence inherent in these species. As well, our approach for isolate genetic relatedness was not robust and we recommend studies with superior approaches such as whole genome sequencing.

\section{Conclusions}

Enterococci are frequent in clinical specimens at Mulago National Referral Hospital but they are susceptible to antibiotics commonly used to treat their infections e.g. ampicillin. As well, the frequency of MDR enterococci in the hospital is low and VRE isolates of E. faecium and $E$. faecalis are rare. However, non-faecium and non-faecalis VRE (E. gallinarum/casseliflavus) are frequent some with VanA and VanB high-level resistance to vancomycin. Thus, species-level identification of VRE isolates is important for instituting effective infection control measures that will curb the spread of VRE in the hospital.

\section{Additional files}

Additional file 1: Table S1. Samples processed for isolation of bacteria. (DOCX $14 \mathrm{~kb}$ )

Additional file 2: Figure S1. Representative images (1\% agarose gels) showing PCR detection of the vanA \& vanB genes. vanA/vanB positive VRE isolates (E. casseliflavus/gallinarum) possessed the expected PCR product sizes i.e. $677 \mathrm{bp}$ and $463 \mathrm{bp}$ for $\operatorname{vanA}$ and $\operatorname{vanB}$, respectively. Lanes in panel A depict: Lad, 100 bp ladder; Pos \& Neg, vanA positive \& negative controls, respectively; $1-7$, samples of which $1,2,5,6,7$ \& 8 were vanA gene-positive while $3 \& 4$ were negative. Lanes in panel $B$ depict: Lad, 100 bp ladder; Pos \& Neg, vanB positive \& negative controls, respectively; $1-7$, samples of which $1,2, \& 3$ were van $B$ gene-positive while $4 \& 5$ were negative $(6 \& 7$ are repeats of $2 \& 3$ ). (TIFF $5313 \mathrm{~kb}$ )

\section{Abbreviations}

AMP: Ampicillin; AST: Antimicrobial susceptibility testing; BEA: Bile Esculin Agar; BHI: Brain heart infusion broth; CIP: Ciprofloxacin; DAP: Daptomycin; ERY: Erythromycin; GEN: Gentamicin; HGT: Horizontal gene transfer; ID: Identification; LIZ: Linezolid; MDR: Multidrug resistance; MIC: Minimum inhibitory concentration; MXF: Moxifloxacin; NIT: Nitrofurantoin; PCR: Polymerase chain reaction; SSI: Surgical site infection(s); TEC: Teicoplanin; TET: Tetracycline; UTIs: Urinary tract infections; VAN: Vancomycin; VRE: Vancomycin resistant enterococci

\section{Acknowledgements}

We thank Mr. Moses Okee for helping with cluster analysis of the rep-PCR assays.

\section{Authors' contributions}

DPK \& CFN conceived, designed and supervised the study, and they participated in data collection and analysis. ME performed the laboratory procedures and data analysis in partial fulfilment of the requirements for the award of the degree of Master of Science in Molecular Biology \& Biotechnology of Makerere University. EK supervised the molecular assays while JK and HB supervised culturing and AST/ID. FNM participated in study supervision. DPK \& CFN wrote the manuscript. All authors read and approved the final version of the manuscript.

\section{Funding}

This study was supported in part by the GlaxoSmithKline (gsk) Trust in Science funding scheme (to DPK). The funder had no role in study design nor decision on where to publish the manuscript.

Availability of data and materials

All data generated or analyzed during this study are included in this published article [and its additional files].

\section{Ethics approval and consent to participate}

Ethical approval to use archived isolates for the study was sought from the Institutional Review Board (IRB), School of Biomedical Sciences, College of Health Sciences, Makerere University. The ethics committee waived the requirement for informed consent as the investigated isolates were obtained from clinical specimens referred to the diagnostic laboratory as part of routine care.

Consent for publication

Not applicable

\section{Competing interests}

The authors declare that they have no competing interests.

\section{Author details}

${ }^{1}$ Department of Immunology and Molecular Biology, Makerere University College of Health Sciences, Kampala, Uganda. ${ }^{2}$ Department of Biomolecular Resources \& Biolab Sciences, School of Biosecurity, Biotechnical \& Laboratory Sciences, College of Veterinary Medicine, Animal Resources and Biosecurity, Makerere University, Kampala, Uganda. ${ }^{3}$ Department of Medical Microbiology, Makerere University College of Health Sciences, Kampala, Uganda. 
Received: 14 February 2019 Accepted: 28 May 2019

Published online: 31 May 2019

\section{References}

1. Tremblay $C L$, Letellier A, Quessy S, Boulianne M, Daignault D, Archambault M. Multiple-antibiotic resistance of Enterococcus faecalis and Enterococcus faecium from cecal contents in broiler chicken and Turkey flocks slaughtered in Canada and plasmid colocalization of tetO and ermB genes. J Food Prot. 2011;74(10):1639-48.

2. Kayser FH. Safety aspects of enterococci from the medical point of view. Int J Food Microbiol. 2003:88(2):255-62.

3. Hasani A, Sharifi Y, Ghotaslou R, Naghili B, Hasani A, Aghazadeh M, Milani M, Bazmani A. Molecular screening of virulence genes in high-level gentamicin-resistant Enterococcus faecalis and Enterococcus faecium isolated from clinical specimens in Northwest Iran. Indian J Med Microbiol. 2012;30(2):175-81.

4. Eliopoulos GM, Gold HS. Vancomycin-resistant enterococci: mechanisms and clinical observations. Clin Infect Dis. 2001;33(2):210-9.

5. Britt NS, Potter EM. Clinical epidemiology of vancomycin-resistant Enterococcus gallinarum and Enterococcus casseliflavus bloodstream infections. Journal of global antimicrobial resistance. 2016;5:57-61.

6. Reid KC, Cockerill IIIFR, Patel R. Clinical and epidemiological features of Enterococcus casseliflavus/flavescens and Enterococcus gallinarum bacteremia: a report of 20 cases. Clin Infect Dis. 2001:32(11):1540-6.

7. Lindenstrauss AG, Ehrmann MA, Behr J, Landstorfer R, Haller D, Sartor RB, Vogel RF. Transcriptome analysis of Enterococcus faecalis toward its adaption to surviving in the mouse intestinal tract. Arch Microbiol. 2014;196(6):423-33.

8. Mwaka AD, Mayanja-Kizza H, Kigonya E, Kaddu-Mulindwa D. Bacteriuria among adult non-pregnant women attending Mulago hospital assessment Centre in Uganda. Afr Health Sci. 2011;11(2):182-9.

9. Seni J, Najjuka CF, Kateete DP, Makobore P, Joloba ML, Kajumbula H, Kapesa A, Bwanga F. Antimicrobial resistance in hospitalized surgical patients: a silently emerging public health concern in Uganda. BMC Research Notes. 2013;6(1):298

10. Teixeira LM, Merquior VLC. Enterococcus. In: Molecular Typing in Bacterial Infections. Edn. New York: Springer; 2013. p. 17-26.

11. Lu JJ, Perng CL, Chiueh TS, Lee SY, Chen CH, Chang FY, Wang CC, Chi WM. Detection and typing of vancomycin-resistance genes of enterococci from clinical and nosocomial surveillance specimens by multiplex PCR. Epidemiol Infect. 2001;126(3):357-63.

12. Kateete DP, Nakanjako R, Okee M, Joloba ML, Najjuka CF. Genotypic diversity among multidrug resistant Pseudomonas aeruginosa and Acinetobacter species at Mulago Hospital in Kampala, Uganda. BMC Research Notes. 2017;10(1):284.

13. Mendiratta DK, Kaur H, Deotale V, Thamke DC, Narang R, Narang P. Status of high level aminoglycoside resistant Enterococcus faecium and Enterococcus faecalis in a rural hospital of Central India. Indian J Med Microbiol. 2008; 26(4):369-71.

14. Gangurde N, Mane M, Phatale S. Prevalence of multidrug resistant enterococci in a tertiary care hospital in India: a growing threat. Open Journal of Medical Microbiology. 2014:4(1):11.

15. Abamecha A, Wondafrash B, Abdissa A. Antimicrobial resistance profile of Enterococcus species isolated from intestinal tracts of hospitalized patients in Jimma, Ethiopia. BMC research notes. 2015;8:213.

16. Kannangara C, Chandrasiri P, Corea EM. Vancomycin resistant enterococcal (VRE) colonization among patients treated in intensive care units at the National Hospital of Sri Lanka, and determination of genotype/s responsible for resistance. The Ceylon medical journal. 2018;63(4):154-8.

17. Desai PJ, Pandit D, Mathur M, Gogate A. Prevalence, identification and distribution of various species of enterococci isolated from clinical specimens with special reference to urinary tract infection in catheterized patients. Indian J Med Microbiol. 2001;19(3):132-7.

18. Landete JM, Peiroten A, Medina M, Arques $J$, Rodriguez-Minguez E. Virulence and antibiotic resistance of enterococci isolated from healthy breastfed infants. Microbial drug resistance (Larchmont, NY). 2018;24(1):63-9.

19. Kateete DP, Kabugo U, Baluku H, Nyakarahuka L, Kyobe S, Okee M, Najjuka CF, Joloba ML. Prevalence and antimicrobial susceptibility patterns of Bacteria from milkmen and cows with clinical mastitis in and around Kampala, Uganda. PLoS One. 2013:8(5):e63413.

20. Osoba AO, Jeha MT, Bakheshwain S, Al Anazi K, Bartlett F. Septicaemia due to vancomycin resistant enterococcus: a case report. Saudi medical journal. 1995;16(1):67-9.

\section{Publisher's Note}

Springer Nature remains neutral with regard to jurisdictional claims in published maps and institutional affiliations.

\section{Ready to submit your research? Choose BMC and benefit from:}

- fast, convenient online submission

- thorough peer review by experienced researchers in your field

- rapid publication on acceptance

- support for research data, including large and complex data types

- gold Open Access which fosters wider collaboration and increased citations

- maximum visibility for your research: over $100 \mathrm{M}$ website views per year

At $\mathrm{BMC}$, research is always in progress.

Learn more biomedcentral.com/submissions 